Facultad de Ciencias Naturales y Exactas

Universidad del Valle

\title{
Reflections on the Difficulties that the Development and Progress of Organic Metallic Chemistry have in Colombia
}

\author{
Esperanza Galarza de Becerra \\ Universidad del Valle \\ Received: May 22, $2014 \quad$ Accepted: Auguts 12, 2014
}

\begin{abstract}
This paper discusses the relatively slow development of research on organometallic chemistry in Colombia compared to that in the world, based on funded projects and publications, as well as, government and educational issues. This paper also discusses the need to promote developments in this area, highlighting the importance of basic scientific research. Its aim is to motivate peers to lead educational processes and research through networking, collaboration and cooperation programs at the national and international level.
\end{abstract}

Keywords: Organometallic Chemistry in Colombia, basic scientific research.

\section{Algunas reflexiones acerca de las dificultades del desarrollo y auge de la química organometálica en Colombia}

\section{Resumen}

Este es un artículo de reflexión en el que se pretende ilustrar el poco auge y desarrollo de la química organometálica en Colombia en comparación con el mundo. Esto se hizo con base en proyectos y publicaciones, aspectos gubernamentales y pedagógicos. El propósito de esta reflexión es resaltar la necesidad de apoyar esta área, subrayando la importancia de la investigación científica y motivar a los pares a liderar procesos pedagógicos e investigativos a través de redes, asociaciones y convenios a nivel nacional e internacional.

Palabras clave: Química organometálica, Ciencia básica.

\section{Introducción}

En este artículo se ilustra sobre la definición e importancia de la investigación básica en la química organometálica y en sus aplicaciones; se plantean dificultades desde los aspectos de grupos de investigación, dificultades para publicar, aspectos experimentales inherentes al área, la falta de apoyo gubernamental, los aspectos pedagógicos, además de unas conclusiones y apreciaciones generales. 


\section{A cerca de la química organometálica en Colombia}

Con relación al tema, la química organometálica como su nombre lo indica, es el área donde la química orgánica e inorgánica se articulan. Se considera un compuesto organometálico un material en el cual hay un enlace iónico o covalente, localizado o deslocalizado, entre uno o más átomos de carbono de un grupo o molécula orgánica con un(os) átomo(s) metálico(s) que puede(n) ser del grupo principal, de transición, lantánidos o actínidos en general.

En la investigación básica, en el marco de la química organometálica, juega un papel importante el estudio de conceptos tales como estructura atómica, enlaces químicos, propiedades químicas, química de superficies, compuestos con estructuras diversas e inusuales, entre otros, conocimiento que ha llevado a la obtención de compuestos con aplicaciones en procesos catalíticos a escala industrial, en fase homogénea o heterogénea, síntesis fina de moléculas orgánicas, en medicina, desarrollo de nuevos materiales, nanociencia y nanotecnología.

Las aplicaciones en medicina han llevado a los científicos a desarrollar nuevos complejos organometálicos, por ejemplo, de platino(II), pioneros en la síntesis de anticancerígenos basados en metales de transición, entre los que se encuentran Eloxatin ${ }^{\circledR}$, utilizado en el tratamiento del cáncer colorrectal, y Satraplatin, usado en el tratamiento del cáncer de próstata. Además de platino, también hay reportes de estudios hechos con complejos organometálicos de rutenio para tratar diversos tipos de cáncer [1].

También se tienen reportes de complejos de Ni(II) con ligandos derivados del triazol con actividad antifúngica [2], así como el uso de compuestos de Au(III) en el tratamiento de la artritis [3]; por otro lado, los seres vivos utilizan cantidades traza de estos elementos para sus funciones biológicas, como es el caso del Zn, elemento presente en el centro activo de la enzima anhidrasa carbónica, cuya función primaria en animales es interconvertir el dióxido de carbono y el bicarbonato para mantener el equilibrio ácido-base en la sangre y otros tejidos, y ayudar al transporte de dióxido de carbono fuera de los tejidos; el $\mathrm{Cr}$, que potencializa la acción de la insulina, etc., lo que ha dado lugar a otra rama de estudio denominada Bioinorgánica, cuyos avances evidencian la participación de numerosas enzimas que contienen centros metálicos y forman compuestos organometálicos en algunos pasos de los procesos de catálisis enzimática. La naturaleza ha estado utilizando sistemas organometálicos para mantener la vida desde hace mucho tiempo, como, por ejemplo, la vitamina B12.

Tradicionalmente, los compuestos organometálicos sintetizados en el laboratorio han sido diseñados principalmente para procesos industriales y catálisis, ahora se les está dando otro enfoque debido a que las investigaciones realizadas en diferentes ámbitos dejan en claro que los compuestos organometálicos tienen potenciales aplicaciones en biología y medicina, como sensores y agentes terapéuticos, como ya se mencionó. Es un reto ahora, también, evidenciar el mecanismo por el cual un compuesto organometálico ejerce un efecto particular, en aspectos medicinales o de otro tipo, en un entorno tan complicado como una célula viva. Además, se han establecido vínculos con la química de materiales ya que los compuestos organometálicos (y metalo-orgánicos) se han convertido en los precursores 
favoritos para la deposición de materiales sobre varios sustratos, por descomposición térmica del compuesto metálico. Por último, la nanociencia y la nanotecnología también se han beneficiado de la química organometálica, pues esta clase de compuestos son los precursores ideales para la síntesis de nanopartículas $[4,5]$.

En el afán por comprender el desarrollo de la investigación en química organometálica es fundamental examinar el peso de los grupos que trabajan en la investigación global en química. La literatura reporta en las últimas décadas múltiples y diversos ejemplos del auge de la química organometálica a nivel mundial; sin embargo, lastimosamente no sucede lo mismo en Colombia.

Según la plataforma ScienTI, de Colciencias [6], en Colombia hay 111 grupos de investigación en diferentes áreas de la química. De estos, puede considerarse que 13 investigan en el ámbito de la química inorgánica, en donde las líneas de investigación con mayor acogida son aquellas que buscan la obtención de nuevos materiales que puedan ser aplicados en catálisis, en su mayor parte heterogénea, o en celdas energéticas. Por el contrario, se observa una gran cantidad de grupos interesados en la química orgánica, la química de nuevos materiales orgánicos, productos naturales (con injerencia de la química farmacéutica) y bioquímica.

Respecto a la química organometálica, en Colombia, de los 13 grupos de investigación en química inorgánica mencionados, 10 pueden considerarse enfocados en la obtención de materiales tales como nanopartículas, polímeros o especies nanoporosas que posteriormente puedan ser aplicados en catálisis heterogénea, entre otros. Solo tres de esos grupos puede decirse que dedican parte de sus proyectos a la obtención de nuevos compuestos químicos con el fin primario de estudiarlos estructuralmente y realizar síntesis per se.

Al observar la Gráfica 1, que describe el estado de los proyectos de química financiados en las diferentes regiones de Colombia, se aprecia que Antioquia, Bogotá, Santander y Valle cuentan con el mayor número de proyectos aprobados, lo que obedece a la presencia de instituciones de educación superior de alto nivel, con un historial científico más sobresaliente que en el resto del país. Es curioso observar que se asignan 3 categorías diferentes relacionadas directamente con química orgánica (síntesis, materiales y compuestos bioactivos), mientras solo hay dos para química inorgánica (síntesis y materiales); lo anterior demuestra claramente el favoritismo por la química orgánica, lo cual no es una tendencia colombiana sino mundial, por las razones ya expuestas en este escrito aunque hay que aclarar que es un área de estudio más reciente. Sin embargo, ambas ramas de la química se encuentran en gran desventaja con respecto a la química de productos naturales, la cual recibe gran apoyo debido a las múltiples materias primas vegetales que en Colombia aún no han sido caracterizadas y cuya aplicación no ha sido descubierta y dentro de la cual también están los resultados de la química farmacéutica.

En la gráfica no solo se observa falta de correspondencia con respecto a las diferentes ramas de la química, sino que también se evidencia la escasez de proyectos aprobados 
en todo el país. En el caso del Valle del Cauca, que cuenta con tres instituciones públicas de educación superior (Universidad del Valle, Universidad Central del Valle del Cauca y Universidad Nacional de Colombia-Sede Palmira), solo han sido aprobados y financiados 23 proyectos en todas las áreas de la química, en donde 4 corresponden a química inorgánica (en sus dos categorías).

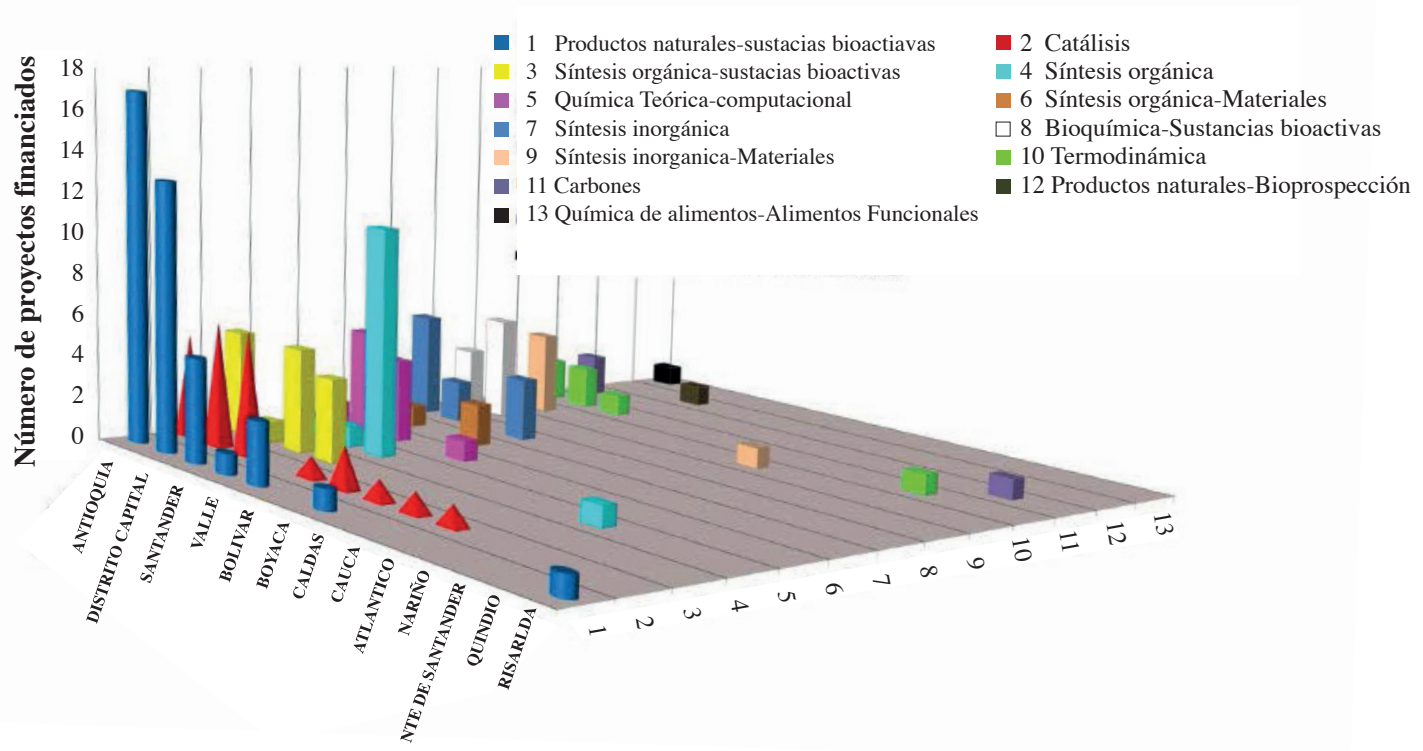

Gráfica 1. Proyectos en química financiados en los diferentes departamentos de Colombia.

Cuando se analiza la base de datos Scopus, Colombia solo muestra 6 publicaciones en química organometálica, mientras países como Brasil y Argentina presentan 193 y 122, respectivamente.

En este examen, juegan un papel importante las publicaciones en esta área investigativa. A continuación se relaciona una serie de datos que permiten clarificar este aspecto, como, por ejemplo, la falta de apoyo por parte del gobierno nacional hacia los proyectos de investigación en química, y más específicamente en química inorgánica y organometálica, el cual se ve reflejado no solo en la escasez de grupos de investigación dedicados a estas ramas de la química, sino también en los desalentadores resultados obtenidos por los grupos activos en cuanto a publicaciones. Según la base de datos Isiweb, de los 8.296 artículos publicados entre 2001 y 2013 en el área de química inorgánica, solo 8 corresponden a Colombia, mientras que de los 5.849 artículos relacionados con la química organometálica en el mismo lapso de tiempo, solo 2 provienen de universidades colombianas. Estas cifras son muy bajas, incluso si se compara con otros países de Latinoamérica, como lo muestra la Gráfica 2. Este comparativo es muy claro al mostrar que Colombia se encuentra muy por debajo de países que destinan un porcentaje considerable del producto interno bruto a investigación. 


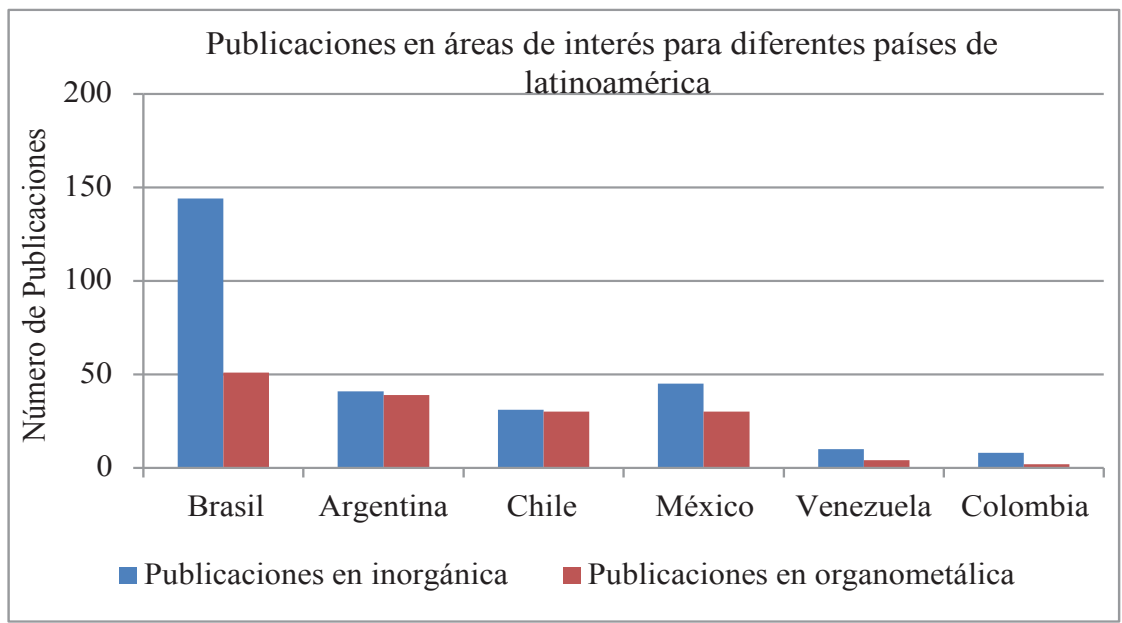

Gráfica 2. Publicaciones en las ramas de la química de interés en Latinoamérica, según Isiweb.

Otro aspecto tiene que ver con la base experimental para la investigación; los altos costos de los reactivos metálicos de partida influyen negativamente en las cantidades utilizadas y en consecuencia repercuten desfavorablemente en el estudio de las actividades y en la caracterización completa de los productos. A esto se suma la dificultad de trabajar en ambientes ausentes de oxígeno y los altos costos de equipos, líneas y cámaras de vacío, usadas para el desarrollo de reacciones bajo atmósfera inerte. Así mismo, procesos de purificación de solventes y reactivos totalmente anhidros. Las propiedades metálicas exigen un gran cuidado y destreza por parte de los investigadores, así como equipos e instrumentos especializados y manejo de técnicas sofisticadas de purificación y caracterización.

Se podría decir que las mismas dificultades existen en otras latitudes; sin embargo, obviando los problemas experimentales propios de la investigación, para el desarrollo de la investigación en química organometálica es prioritario el apoyo estatal, pero eso no sucede en Colombia. No se puede dejar de hablar de la política utilizada por Colciencias desde hace varios años para aprobar los proyectos que se presentan a concurso. La tendencia es aprobar proyectos que tengan aplicaciones inmediatas, desconociendo la importancia de la investigación en ciencias básicas. Parte de la investigación en química debe dedicarse a tratar de entender la estructura y cambios de la materia, siendo esta la única forma para obtener una mayor eficacia al inferir sus posibles aplicaciones. Los proyectos financiados por Colciencias en química inorgánica han sido 16 desde 1991, según el Consejo Extraordinario Nacional de Ciencias Básicas, realizado en 2013. Si existieran datos para los proyectos financiados en la sub-área de química organometálica, esta cifra seguramente sería muy desconcertante porque disminuiría aún más.

Por esta razón, se cuestionan las estrategias usadas por esta entidad estatal, financiadora de proyectos para la mejora de la calidad investigativa en Colombia, y seguramente de nada servirá seguir permitiendo que el número de grupos de investigación aumente si no se les aprueban proyectos y recursos. De igual manera, será muy complicado llevar un proyecto a buen término si en las universidades o centros de investigación no se tiene la tecnología necesaria para realizar los estudios y análisis pertinentes. Además, las cifras de publicaciones demuestran que tanto en el caso de la química inorgánica como en la organometálica, el enfoque de apoyo a proyectos con aplicación inmediata no ha surtido 
Aunque la estrategia de semilleros y jóvenes investigadores implementada por Colciencias es acertada, debe aplicarse con mayor cobertura hacia aquellos grupos que se están iniciando o que necesitan recursos más específicos y costosos para desempeñarse debidamente.

Otro problema es la relación entre formación e investigación y surgirían muchos interrogantes como, por ejemplo: ¿Qué condiciones se deben cumplir en el proceso formativo para que los estudiantes investiguen el área de química organometálica?

Al hacer un enfoque hacia el interior del programa de Química de la Universidad del Valle, se nota el esfuerzo para la adecuación y mejora de los laboratorios de docencia, de los instrumentos, insumos y equipos básicos, cuyo acceso requiere un examen calificativo previo, entre otros. Suponiendo que los estudiantes posean buenas herramientas conceptuales y los temas estén bien afianzados, para realizar buenas prácticas de laboratorio en química inorgánica hace falta apoyo tecnológico. En tal consideración, aunque la temática de la Química Inorgánica I y II es amplia, es indudable que se requiere un nivel más de química inorgánica. Se presupone que lo mismo sucede con la carrera de Química de las otras universidades nacionales y no se entiende por qué se ha insistido durante tantos años en no poner en igualdad de créditos a la química orgánica e inorgánica.

Es importantísimo, también, revisar la pedagogía experimental en inorgánica, y hacer un cambio de enfoque de las prácticas de laboratorio en inorgánica. Como lo han mencionado varios investigadores de pedagogía científica, debería proponerse como objetivo de la enseñanza experimental ilustrar acerca del proceso mediante el cual se hace ciencia, en vez de hacer que el estudiante aprenda procedimientos o atienda instrucciones acerca de qué tipo de datos obtener y cuándo tomar las medidas; luego, pedirle que organice tales datos en una tabla y, por último, decirle paso a paso qué cálculos y análisis debe realizar para obtener una conclusión [7]; la realización aislada y sin ilación de estas actividades no genera estudiantes con la capacidad de diseñar, de principio a fin, una metodología para obtener resultados en una investigación en inorgánica, ni personas independientes y responsables, sino que los mecaniza acerca de qué es lo que se supone deben hacer al llegar al laboratorio, así no sepan por qué o para qué.

El nuevo sistema pedagógico en boga es denominado Inquiry-Based Instruction (Estrategia de Instrucción Basada en la Interrogación) [8], y trata de enseñar una metodología basada en la construcción, afrontando las prácticas de laboratorio como ayudas para resolver problemas y preguntas, que es lo que realmente sucede en la investigación [4]. Se tienen reportes de haber aplicado este método en prácticas de química inorgánica [8-11]; sin embargo, algunos de los equipos utilizados para caracterizar (difracción de rayos X para polvos, susceptibilidad magnética, fotosensibilidad, espectrometría de masas, entre otros) son bastante especializados y la mayoría de las universidades públicas de Colombia, o no cuentan con el equipo, o cuentan con solo uno (o un escaso número) que es destinado preferiblemente a labores investigativas y no de docencia.

Todos los puntos anteriormente expuestos demuestran la necesidad de apoyar más esta área de la química, de brindarle recursos y espacios en los programas de Química de las universidades públicas colombianas. Para mejorar la investigación en química inorgánica y organometálica en Colombia, para permitir que las cifras en artículos publicados y el 
descubrimiento de nuevas aplicaciones para los compuestos descubiertos sean posibles, es necesario revisar muchos de los aspectos planteados en esta reflexión preliminar y otros como la búsqueda de financiación externa y la formación de redes nacionales e internacionales.

La reflexión plantea un compromiso que se deriva de la importancia de la investigación en compuestos organometálicos y que conlleva a plantear en los profesores del área el deseo por liderar redes y agrupaciones, siendo este un desafío para las nuevas generaciones, el cual debe ser afrontado con entusiasmo, amor y responsabilidad, llevando a cabo investigaciones rigurosas y, en algunos casos, netamente académicas de los investigadores actuales, actores de la investigación y docencia en química organometálica.

\section{Referencias bibliográficas}

[1] Antonarakis, E. S., Emadi, A. (2010). Ruthenium based chemotherapeutics: are they ready for prime time? Cancer Chem. Ther. Pharmacol., 66, 1-9.

[2] Zabin, S. A. (2011). Synthesis and Antimicrobial Studies of Cu(II), Ni(II) and Zn(II) Schiff Base Complexes Derived from Substituted 1,2,4-Triazoles and Heteroaromatic Aldehydes. Asian. J. Chem. 23, 4067-4071.

[3] Anilanmert B. (2012). Therapeutic Organometallic Compounds, Pharmacology, Dr. Luca Gallelli (Ed.), ISBN: 978-953-51-0222-9, InTech, DOI: 10.5772/33858.

[4] Crabtree, R. H. (2005). The organometallic chemistry of transition metals. New Jersey: John Wiley \& Sons, Inc.

[5] Hill, A. F. (2002). Organotransition Metal Chemistry. Cambridge: The Royal Society of Chemistry.

[6] Colciencias (2014). Recuperado de: http://scienti.colciencias.gov.co:8083/cienciawar/BusquedaGrupoXArea.do revisado 23-10-2014.

[7] Carriazo, J. G. (2011). Laboratory projects using inquiry-based learning: an application to a practical inorganic course. Quim. Nova, 34 (6), 1085-1088.

[8] Ludwin, V. (2000). Inquiry-based experiments in chemistry. American Chemical Society, Washington.

[9] Carriazo, J., M. Saavedra (2004). Chemical Education: An Arising Research Field. Tecné, Episteme y Didaxis, 15, 73-84.

[10] Carriazo, J., D. Pérez-Sotelo, A. Ensuncho-Muñoz (2005). Synthesis and Characterization of Coordination Compounds: An Experience of Learning by Research in Inorganic Chemistry. Tecné, Episteme y Didaxis, 18, 34-46.

[11] Carriazo, J. G. (2010). Inquiry-based experiment on the synthesis of $\mathrm{K}_{3}\left[\mathrm{Fe}\left(\mathrm{C}_{2} \mathrm{O}_{4}\right)_{3}\right] \cdot 3 \mathrm{H}_{2} \mathrm{O}$. Chemistry, 19 (4), 103-112.

\section{Dirección de la autora}

Esperanza Galarza

Departamento de Química, Universidad del Valle, Cali - Colombia.

esperanza.galarza@correounivalle.edu.co 